\title{
Evaluation of the applicability of bioimpedance in the quantification of body fluid compartments
}

\author{
Avaliação da aplicabilidade da bioimpedância à quantificação dos compartimentos \\ fluidos corporais
}

\author{
Lídia Palma, Ana Rita Alves, Liliana Tavares, Carla Monteiro \& Luis Monteiro Rodrigues \\ CBIOS - Research Center for Health Science and Technologies, Universidade Lusófona, Campo Grande, \\ 376, 1749-024, Lisboa, Portugal \\ Email: monteirorodrigues@sapo.pt
}

\begin{abstract}
Bioimpedance seems to be particularly suited to the non-invasive measurement of several physiological variables. The present study aimed to ascertain whether the quantification of total body water and intracellular and extracellular fluid compartments, obtained through a bioimpedance-based analytical device, could be correlated with the concentration sodium, potassium and chloride ions, obtained from venous blood ionogram. Volunteers of both genders, of various ages and with various health conditions were studied. None of the volunteers suffered from any hydro-electrolytic disorder. Body water measurements were obtained by a Bodystat ${ }^{\circledR}$ QuadScan 4000 and ionogram obtained through potentiometry. Under the present experimental conditions, which should be considered merely a preliminary approach to the questions raised, no relations were established between the variables under study. However, the method's potential interest and its practicability justifies further investigation. This would include increasing the number of patients studied and refining the methodology used.
\end{abstract}

Keywords: bioimpedance; body water; natremia; kalemia; ionogram.

\section{Resumo}

A bioimpedância parece ser especialmente adequada à medição não-invasiva de diversas variáveis fisiológicas. $\mathrm{O}$ presente estudo pretendeu averiguar se a quantificação da água corporal total e dos compartimentos intra e extracelular, obtida com um equipamento de análise baseada na bioimpedância, é relacionável com os valores das concentrações dos iões sódio, potássio e cloreto obtido a partir do ionograma do sangue venoso. Foram estudados 12 voluntários, de ambos os géneros, idade e condição de saúde variadas, embora sem qualquer patologia do foro hidro-electrolítico. As medições da água corporal foram obtidas com o Bodystat ${ }^{\circledR}$ QuadScan 4000 e o ionograma por potenciometria. Nas presentes condições experimentais, que apenas devem ser entendidas como uma abordagem preliminar às questões de investigação levantadas, não foram demonstradas relações entre as variáveis em estudo. Contudo, o interesse potencial e a praticabilidade do método, justificam novas abordagem que visem aumentar o número de paciente estudados e refinar a metodologia de análise.

Palavras-chave: bioimpedância; agua corporal; Natrémia, Potassémia, ionograma; 


\section{Introduction}

Several diagnostic and therapeutical techniques based on so-called bioimpedance analysis have been widely used in human healthcare. Such is the case of the electrocardiogram (ECG), the electroencephalogram (EEG) and, more recently, the iontophoresis (electrotherapy for pain relief) and electroporation. These techniques are of remarkably easy and quick applicability and operation, are non- or minimally Invasive, are very well-tolerated by patients and are quite reliable, despite known difficulties ${ }^{[1,2]}$

The electrical behavior of the human body's tissues is still not well known. It is accepted that the electrical current frequency (in Hertz, Hz), and its type (alternate or direct), have an effect on its passage through the cells reaching each tissue.

Bioimpedance analysis has become an especially interesting method for measuring other hard-to-tackle variables, such as water in the body fluid compartments (intracellular and extracellular) which are variables of critical importance in kidney and hydro-electrolytic disorders. Reference methods are mostly impractical, based on hydrostatic weighing by the Archimedes' principle -, through which body density and body composition, including fat mass, are obtained ${ }^{[2,5-9]}$. The administration of stable radioactive elements per os such as ${ }^{2} \mathrm{H}$ and ${ }^{18} \mathrm{O}$, though also important, is hardly applicable ${ }^{[10-12]}$. Blood sodium bromide $(\mathrm{NaBr})$ determination ${ }^{[13,14]}$, air displacement plethysmography ${ }^{[15]}$ and bone densitometry (DEXA Dual-Energy X-ray Absorptionmetry) ${ }^{[16]}$ are other sophisticated methods used with the same purpose.

This recently available bioimpedance system - the Bodystat ${ }^{\circledR}$ QuadScan 4000 - is apparently able to non-invasively quantify the body water along with other variables related to the distribution of the body fat (the tissue with the lowest amount of water) referred to as the fat mass, and the muscular tissue, referred to as the lean mass..The present study preliminarily addresses the reliability of these data.

\section{Material and Methods}

12 healthy volunteers of both genders $(6$ men and 6 women), aged between 21 and $82(37.7+18.6)$ y.o. were selected from the General Medicine patients of the São João de Deus Health Care Group and were included after a detailed explanation of the purpose and

\section{Introdução}

Diversas técnicas de diagnóstico e terapêuticas baseadas na avaliação da chamada bioimpedância têm sido amplamente utilizadas em saúde humana, como é o caso do electrocardiograma (ECG) e do electroencefalograma (EEG), e mais recentemente da iontoforese (electroterapia para alívio da dor), e da electroporação. São reconhecidamente técnicas de fácil e rápida aplicabilidade e operação, minimamente invasivos ou mesmo não-invasivos, muito bem toleradas pelos pacientes, e bastante fiáveis, apesar das dificuldades que o tema encerra ${ }^{[1,2]}$.

O comportamento eléctrico dos vários tecidos do corpo humano ainda não é bem conhecido, mas sabe-se que a frequência da corrente eléctrica (medida em Hertz, Hz) e o seu tipo (alterna ou contínua) influencia a sua passagem através das células que constituem qualquer tecido $^{[1]}$.

A análise da bioimpedância tem surgido como um método de especial interesse para a medição de outras variáveis fisiológicas de difícil abordagem, como é o caso da água corporal dos compartimentos fluidos corporais (intra e extra-celulares), especialmente importante em patologia renal e hidro-electrolítica ${ }^{[1-4]}$. Os métodos de referência, são pouco práticos, e baseiam-se na pesagem hidrostática, aplicando o princípio de Arquimedes, através dos quais se obtêm a densidade corporal e composição corporal, incluindo a massa gorda ${ }^{[2,5-9]}$. A administração de elementos radioactivos estáveis por via oral como sejam o ${ }^{2} \mathrm{H}$ e o ${ }^{18} \mathrm{O}$ são também importantes, embora de difícil aplicabilidade ${ }^{[10-12]}$. O doseamento do brometo de sódio (NaBr) no sangue ${ }^{[13,14]}$, a pletismografia por deslocação de ar (ADP Air Displacement Plethysmography) ${ }^{[15]}$, e a densitometria óssea (DEXA Dual-Energy X-ray Absorptionmetry) (16) são ainda outros métodos, sofisticados, utilizados com aquelas finalidades.

Face à recente introdução no mercado de um sistema de bioimpedância alegadamente capaz de permitr a quantificação, por meio não invasivo, não só da àgua corporal mas ainda de outras informações relacionadas com a distribuição corporal de gordura (o tecido com menos àgua) - a massa gorda, e de tecido muscular - a chamada massa magra, o Bodystat ${ }^{\circledR}$ QuadScan 4000, o presente trabalho aborda, ainda que de forma preliminar, a fiabilidade dos dados obtidos.

\section{Materiais e métodos}

Foram seleccionados 12 voluntários saudáveis, de ambos os géneros ( 6 homens e 6 mulheres), de idades compreendidas entre os 21 e os 82 anos $(37,7 \pm 18,6$ anos) de entre os utentes do São João de Deus - Grupo de Saúde da consulta de medicina geral, incluídos após 
objectives of the study, following previously defined inclusion criteria. These volunteers greatly vary in age, diet, physical and health conditions. Hypertension and hypercholesterolemia were the most reported health problems by the volunteers. Some declared that they considered themselves as healthy. This variability is critical for any validation purpose, as in the present case. Pregnancy and the existence of any electronic equipment such as a cardiac pacemaker, were considered as non-inclusion criteria. Some restrictions were imposed regarding eating or drinking in the six hours prior to the measurements, physical exercise during the twelve hours before, and drinking alcohol or caffeinated beverages within twenty-four hours before measurements ${ }^{[4]}$. All participants were informed of the study circumstances, conducted in accordance with the Declaration of Helsinki and respective amendments ${ }^{[17,18]}$, and all signed their informed consent.

Volunteers were weighed with an analytical balance (model YHB0401, Kunft, Germany) and their height was measured. The BMI (body mass index) was calculated according to the formula BMI $=$ weight $(\mathrm{kg})$ $/$ height $^{2}(\mathrm{~m})$. Venous blood samples were obtained for analytical routine, which covered ionogram. Sodium, potassium and chloride concentration values were determined with a potenciometric ion-selective electrode (ISE-8860, and 8862-ISE ISE-8760, Omega Engineering, USA) ${ }^{[6.19]}$. All analytical chemistry was performed at the Nova Era Luz Laboratory (São João de Deus-Health Care Group, Lisbon).

Body water measurements were obtained by a Bodystat QuadScan 4000 ( $)$ unit (Bodystat - Isle of Man, UK) which operates with several frequencies allowing us to obtain Total Body Water (TBW Total Body Water) at $200 \mathrm{kHz}$ and Extracellular Water (ECW Extracellular Water) at $5 \mathrm{kHz}$. The Intracellular Water compartment values (ICW Intracellular Water) are obtained indirectly, by the difference between the values of TBW and ECW. The Bodystat QuadScan ${ }^{\circledR}$ 4000 further calculates TBW at $50 \mathrm{kHz}$. The difference between these two TBW values is a variable referred to as "water on the third space,". This is not relevant to the circumstances of the present study, except in situations where its value exceeds the 2 litres which would indicate edema ${ }^{(4)}$. The device uses a previously developed and published regression equation. ${ }^{[2,20]}$

For biometric measurements the patient is positioned in dorsal decubitus and electrodes placed in the right upper limb (one immediately below the proximal phalanges, another under the radiocubital joint) and in the left lower limb (one immediately below the proximal phalanges, the other in the ankle joint), (Figure 1), according to the manufacturer's specifications $^{[2]}$. explicação detalhada do propósito e objectivos do estudo, considerando os critérios de inclusão antes definidos. Estes voluntários constituem uma amostra extremamente variada no que respeita à idade, aos regimes alimentares e condição física, bem como às suas condições de saúde. Hipertensão arterial e hipercolesterolémia foram as patologias pré-existentes mais reportadas pelos voluntários, havendo, no entanto, voluntários que se declararam completamente saudáveis. Esta variabilidade é sobretudo essencial para efeitos de validação de uma metodologia, pelo que foi já aqui considerada. A gravidez, e a existência de qualquer equipamento electrónico implantável como um pacemaker cardíaco foram adoptados como critérios de não-inclusão. Foram ainda impostas algumas restrições, como não beber ou comer nas seis horas anteriores às medições, não praticar exercício físico nas doze horas anteriores e, não ingerir bebidas alcoólicas ou cafeinadas nas vinte e quatro horas anteriores ${ }^{[4]}$. Todos os participantes foram devidamente informados sobre as circunstâncias do estudo, o qual decorreu de acordo com a Declaração de Helsínquia e respectivas emendas ${ }^{[17,18]}$, tendo assinado o respectivo consentimento informado.

Os voluntários foram pesados com uma balança analítica (modelo YHB0401, Kunft, Alemanha), e medidos em altura. Desta informação foi calculado o índice de massa segundo a fórmula IMC $=$ peso $(\mathrm{kg}) /$ altura $^{2}(\mathrm{~m})$. Todos os voluntários foram sujeitos a punção venosa para recolha de sangue destinado a análises de rotina, onde se incluiu o ionograma. Os valores das concentrações dos iões sódio, potássio e cloreto foram determinados por potenciometria utilizando eléctrodos ião-selectivos (ISE-8860, ISE8862 e ISE-8760, Omega Engineering, EUA) ${ }^{[6,19]}$. Toda a química analítica foi efectuada no Laboratório de Nova Era-Luz (São João de Deus - Grupo de Saúde, Lisboa).

O equipamento utilizado para efectuar as medições da àgua corporal foi uma unidade Bodystat ${ }^{\circledR}$ QuadScan 4000, comercializada pela empresa Bodystat (Isle of Man, UK). Este equipamento opera em várias frequências, sendo os valores de Água Corporal Total (TBW Total Body Water) calculados a $200 \mathrm{kHz}$ e os de Água no Compartimento Extracelular (ECW Extracellular Water) calculados a $5 \mathrm{kHz}$. Os valores de Água no Compartimento Intracelular (ICW Intracellular Water) são obtidos indirectamente, pela diferença entre os valores obtidos de TBW e ECW. O Bodystat ${ }^{\circledR}$ QuadScan 4000 calcula ainda TBW a 50 $\mathrm{kHz}$, sendo a diferença entre os dois valores de TBW uma variável referida como "água no terceiro espaço", não relevante para as actuais circunstâncias do estudo, excepto em situações em que o seu valor ultrapasse os 2 litros, ocasião em que se deve considerar a possível existência de edema (4). O aparelho utiliza uma 
equação regressiva antes desenvolvida e publicada ${ }^{[2,20]}$ Antes das medições biométricas, os pacientes foram posicionados em decúbito dorsal, e os eléctrodos colocados no membro superior direito (um imediatamente inferior às falanges proximais, outro sob a articulação radiocubital) e no membro inferior esquerdo (um imediatamente inferior às falanges proximais, outro sob a articulação talocrural), (Figura 1), de acordo com as especificações do fabricante ${ }^{[2]}$.

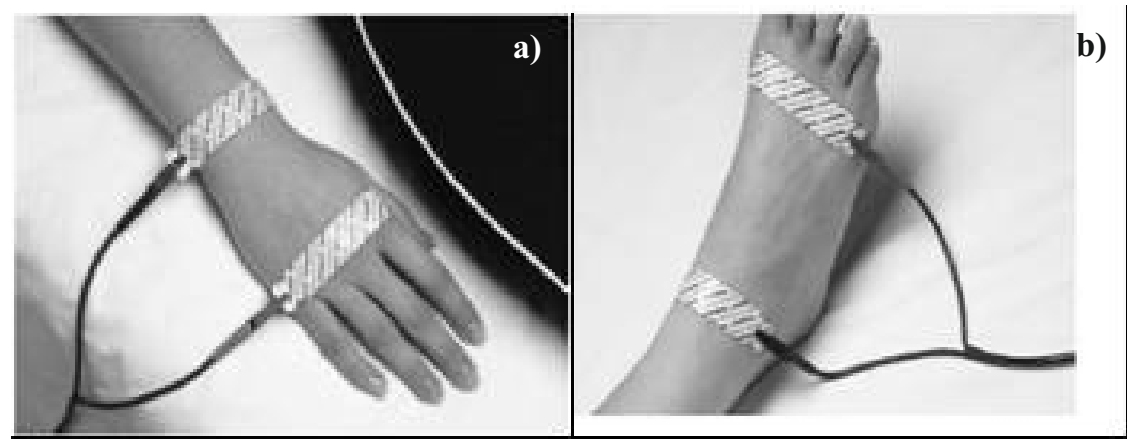

Figure 1 - Illustration about the Bodystat ${ }^{\circledR}$ QuadScan 4000 electrode positioning (check text)

Figura 1 - Ilustração do posicionamento dos eléctrodos utilizados pelo sistema Bodystat ${ }^{\circledR}$ QuadScan 4000 (ver texto).

Statistical analysis was performed with SPSS 20 (IBM, USA). The normality test of Shapiro-Wilk, and in the case of normal distribution, the t-Student, the Pearson's and the Spearman's correlation were used. A range of $95 \%$, and a significance level (p) equal to 0.05 were adopted.

\section{Results and discussion}

Descriptive analysis of the studied variables are shown in Tables 1 to 3 .

Measuring body water as the osmotically relevant electrolytes is an essential part of hydro-electrolyte disorders diagnostics. Hyponatremia and hypernatremia are relatively common situations although difficult to identify and are often associated with more complex multifactorial clinical conditions. For this reason they can be potentially serious ${ }^{[21,22]}$. Total body water (TBW, in Liters), extracellular water (ECW, in Liters) and intracellular water (ICW, in
A análise estatística foi realizada com o software SPSS 20 (IBM, EUA). Foi utilizado o teste de normalidade de Shapiro-wilk e, em caso de distribuição normal, o t de student e a correlação de Pearson. A correlação de Spearman foi também utilizada sempre que adequado. Foram adoptados um intervalo de confiança de $95 \%$, e um nível de significância (p) igual a 0,05.

\section{Resultados e discussão}

A análise descritiva das variáveis em estudo encontrase nas tabelas 1 a 3 .

A medição da água corporal e de electrólitos osmoticamente relevantes é essencial para o diagnóstico da disfunção hidro-electrolítica. A hiponatrémia e a hipernatrémia são situações relativamente comuns, frequentemente difíceis de diagnosticar, muitas vezes associadas a um quadro clínico mais complexo e multifactorial e, por isso, potencialmente grave ${ }^{[21,22]}$. A água corporal total (TBW em Litros), a água no compartimento extracelular 
Liters) were assessed in all volunteers. The ionogram allows us to determine the concentration of sodium $(\mathrm{Na}+)$, potassium $(\mathrm{K}+)$ and chloride $(\mathrm{Cl}-)$ ions, expressed in $\mathrm{mEq} / \mathrm{L}$ in the volunteers serum.
(ECW em Litros) e a água no compartimento intracelular (ICW em Litros) foi determinada em todos os voluntários. O ionograma permitiu obter no soro os valores dos iões sódio $(\mathrm{Na})$, potássio $(\mathrm{K})$ e cloreto $(\mathrm{Cl})$, expressos em $\mathrm{mEq} / \mathrm{L}$.

Table 1 - Descriptive analysis of volunteer's weight (kg), high (m), age and Body Mass Index (BMI) by gender

Tabela 1 - Análise descritiva das variáveis "peso" (kg), "altura" (m) "idade" e Índice de Massa Corporal (IMC) dos voluntários por género.

\begin{tabular}{|c|c|c|c|c|c|c|c|}
\hline & $\mathrm{n}$ & $\begin{array}{c}\text { Range } \\
\text { Intervalo }\end{array}$ & Min & Max & $\begin{array}{c}\text { Mean } \\
\text { Média }\end{array}$ & $\begin{array}{c}\text { Standard } \\
\text { deviation } \\
\text { Desvio padrão }\end{array}$ & Var \\
\hline $\begin{array}{c}\text { Feminine } \\
\text { Feminino }\end{array}$ & 6 & - & - & - & - & - & - \\
\hline $\begin{array}{c}\text { Weight } \\
\text { Peso }\end{array}$ & 6 & 21,50 & 47,80 & 69,30 & 57,10 & 7,30 & 53,00 \\
\hline $\begin{array}{c}\text { High } \\
\text { Altura }\end{array}$ & 6 &, 17 & 1,52 & 1,69 & 1,618 &, 07 &, 00 \\
\hline $\begin{array}{c}\text { Age } \\
\text { Idade }\end{array}$ & 6 & 14 & 21 & 35 & 26,17 & 5,30 & 28,10 \\
\hline $\begin{array}{c}\text { BMI } \\
\text { IMC }\end{array}$ & 6 & 4,70 & 19,60 & 24,30 & 21,75 & 1,92 & 3,70 \\
\hline $\begin{array}{c}\text { Masculine } \\
\text { Masculino }\end{array}$ & 6 & - & - & - & - & - & - \\
\hline $\begin{array}{c}\text { Weight } \\
\text { Peso }\end{array}$ & 6 & 25,80 & 66,40 & 92,20 & 79,17 & 10,13 & 102,70 \\
\hline $\begin{array}{c}\text { High } \\
\text { Altura }\end{array}$ & 6 &, 08 & 1,70 & 1,78 & 1,74 &, 038 &, 00 \\
\hline $\begin{array}{c}\text { Age } \\
\text { Idade }\end{array}$ & 6 & 58 & 24 & 82 & 49,17 & 20,35 & 414,20 \\
\hline $\begin{array}{c}\text { BMI } \\
\text { IMC }\end{array}$ & 6 & 7,00 & 24,50 & 31,50 & 27,63 & 2,60 & 6,80 \\
\hline
\end{tabular}

Table 1 - Descriptive analysis of the volunteer's TBW, ECW and ICW (see text for sigla meaning) Table 2- Análise descritiva das variáveis TBW, ECW e ICW (verifique no texto o significado das siglas)

\begin{tabular}{|l|c|c|c|c|c|c|c|}
\hline & $\mathrm{n}$ & $\begin{array}{c}\text { Range } \\
\text { Intervalo }\end{array}$ & Min & Max & $\begin{array}{c}\text { Média } \\
\text { Mean }\end{array}$ & $\begin{array}{c}\text { Standard } \\
\text { deviation } \\
\text { Desvio padrão }\end{array}$ & Var \\
\hline TBW & 12 & 21,90 & 26,00 & 47,90 & 36,89 & 7,48 & 55,88 \\
ECW & 12 & 7,00 & 13,10 & 20,10 & 16,46 & 2,38 & 5,69 \\
ICW & 12 & 20,10 & 8,30 & 28,40 & 18,27 & 4,62 & 21,39 \\
Valid N (listwise) & 12 & & & & & & \\
\hline
\end{tabular}


Table 3 -Descriptive analysis of the volunteer's variables $\mathrm{Na"}(\mathrm{mEq} / \mathrm{L})$, " $\mathrm{Cl}$ " $(\mathrm{mEq} / \mathrm{L})$ and " $\mathrm{K}$ " $(\mathrm{mEq} / \mathrm{L})$ obtained by ionogram of preiously obtained venous blood samples (see text)

Tabela 3 - Análise descritiva das variáveis "Na" $(\mathrm{mEq} / \mathrm{L})$, " $\mathrm{Cl}$ " $(\mathrm{mEq} / \mathrm{L})$ e " $\mathrm{K}$ " $(\mathrm{mEq} / \mathrm{L})$ obtidos através do ionograma do sangue venoso previamente colhido dos pacientes.(ver texto)

\begin{tabular}{|l|c|c|c|c|c|c|c|}
\hline & $\mathrm{n}$ & $\begin{array}{c}\text { Range } \\
\text { Intervalo }\end{array}$ & Min & Max & $\begin{array}{c}\text { Média } \\
\text { Mean }\end{array}$ & $\begin{array}{c}\text { Standard deviation } \\
\text { Desvio padrão }\end{array}$ & Var \\
\hline $\mathrm{K}$ & 12 & 1,0 & 4,1 & 5,1 & 4,642 &, 3059 &, 094 \\
$\mathrm{Cl}$ & 12 & 6 & 101 & 107 & 103,75 & 1,865 & 3,477 \\
$\mathrm{Na}$ & 12 & 6 & 136 & 142 & 138,75 & 1,658 & 2,750 \\
Valid N (listwise) & 12 & & & & & & \\
\hline
\end{tabular}

Bioimpedance explores the electrical properties of living biological tissues expressing its behavior in terms of impedance which depends on the tissue components, its length and diameter ${ }^{[3]}$. Longer and thinner tissues, with higher water and ions content show lower impedance than shorter, thicker tissues with lower water and ions concentrations ${ }^{[1,11,23]}$. Each tissue impedance seems to depend on the voltage frequency applied and from the water distributed in the intra and extracellular compartments ${ }^{[2]}$. Lower frequencies (up to $100 \mathrm{kHz}$ ) cannot penetrate cell membranes and travel primarily through the extracellular compartment; higher frequencies (higher than $100 \mathrm{kHz}$ ) can penetrate cell membranes and pass through both compartments ${ }^{[1,11]}$. Impedance is the inverse of conductance, being measured in $(\Omega$ $=$ voltage / amperage). Bigger values represent greater difficulties going through the tissue ${ }^{[1]}$. Water and ions are the most important components of those tissues regarded as "lean", as is the case of muscular and nervous tissues as well as extracellular compartment (blood and intersticium) ${ }^{[10]}$. These are highly anisotropic environments with conductance ranging from $10^{-1}$ to $10^{2} \mathrm{~S} / \mathrm{m}$ in muscle and grey matter, respectively, ${ }^{[1]}$, and $0,667 \mathrm{~S} / \mathrm{m}$ in blood ${ }^{[21,24]}$ and with low impedance values ${ }^{[10]}$.

As shown in Table 1 and 2 the variables "weight", height", "age", BMI, TBW, ECW and ICW confirm the group's heterogeneity. Regarding ions $\mathrm{Na}+\mathrm{Cl}-$ and $\mathrm{K}+$ obtained from the venous blood ionogram, all volunteers have shown values within the normal range, showing no sign of any specific hydro-electrolytical disorder (Table 3).

TBW was, among the body water distribution variables, the one showing wider amplitude values. As expected this variable is weight dependent, meaning that bigger weight amplitudes will imply wider variation of TBW. Figure 2 illustrates this relationship 72
A bioimpedância explora as diferentes propriedades eléctricas dos tecidos biológicos cujo comportamento, expresso em termos de impedância, depende do material que compõe o tecido, do seu comprimento e do seu diâmetro ${ }^{[3]}$. Tecidos mais longos e mais finos, contendo maiores quantidades de água e iões, têm menor impedância do que tecidos mais curtos, mais espessos, com pouca água e iões ${ }^{[1,11,23]}$. A impedância de cada tecido parece depender da frequência da voltagem aplicada e da distribuição de água nos compartimentos intra e extracelulares ${ }^{[2]}$. A frequência baixa (até 100 $\mathrm{kHz}$ ) a corrente eléctrica não consegue penetrar as membranas celulares e é conduzida maioritariamente pelo fluido extracelular; a frequências mais altas (superiores a $100 \mathrm{kHz}$ ) a corrente eléctrica já consegue penetrar as membranas atingindo então ambos os compartimentos ${ }^{[1,11]}$. A impedância é o inverso da condutância, e é medida em ohms $(\Omega=$ voltagem/amperagem). Quanto maior o seu valor, mais difícil é para uma corrente eléctrica atravessar esse tecido "[]. Os tecidos considerados "magros", são maioritariamente constituídos por água e iões, como acontece com os tecidos muscular e nervoso, e o compartimento extracelular (sangue e interstício) ${ }^{[10]}$. São meios muito anisotrópicos com valores de condutância, entre $10^{-1}$ e $10^{2} \mathrm{~S} / \mathrm{m}$ no músculo e na massa cinzenta, respectivamente ${ }^{[1]}$, e $0,667 \mathrm{~S} / \mathrm{m}$ no sangue ${ }^{[21,24]}$ e valores de impedância baixos (10).

Como se observa nas Tabelas 1 e 2 as variáveis "peso", altura", "idade", IMC, TBW, ECW e e ICW corroboram a heterogeneidade da amostra seleccionada. No que respeita aos iões $\mathrm{Na}+\mathrm{Cl}-$ e $\mathrm{K}+$ obtidos através do ionograma do sangue venoso observamos que todos os voluntários apresentam valores situados dentro do intervalo de normalidade, não evidenciando qualquer disfunção hidroelectrolítica específica (Tabela 3).

No que respeita às variáveis de distribuição da água 
with a $\mathrm{R}^{2}=0,881$ and a Pearson's correlation coefficient $\left(r=\sqrt{ } \mathrm{R}^{2}\right)$ of 0,939 , a very high value indicating that the weight variation implies a non direct, non reversely proportional variation of TBW. Volunteers with lower weights $(<60 \mathrm{~kg})$ show lower TBW values $(<35 \mathrm{~L})$ (Figura 3$)$

After confirming the normal distribution of these variables, the Pearson's correlation test was applied. This confirmed a strong positive relationship $(\mathrm{Rp}=$ $0,939)$ which was statistically significant $(\mathrm{p}=0,000)$. Lower weights $(<15 \mathrm{~kg})$ correspond to lower TBW values inferior to $35 \mathrm{~L}$. However, due to the lower water content of adipose tissue $(2,25,26)$ it is possible to obtain lower TBW values in heavier patients. corporal, a TBW foi a que apresentou maior amplitude. Conforme esperado, esta variável depende do peso, pelo que a uma maior amplitude de valores do peso corresponderá, previsivelmente, uma maior amplitude de variação de TBW. A figura 2 ilustra esta relação com $\mathrm{R}^{2}=0,881$ e um coeficiente de correlação de Pearson $\left(r=\sqrt{ } R^{2}\right)$ de 0,939 , um valor francamente elevado, sugerindo que a variação do "peso" implicará uma variação não directa nem inversamente proporcional da TBW. Os voluntários com peso mais baixo $(<60 \mathrm{~kg})$ apresentam valores mais baixos de TBW $(<35 \mathrm{~L})$

Confirmada a distribuição normal destas variáveis, aplicámos a correlação de Pearson que demonstrou existir uma relação forte positiva $(R p=0,939)$ e estatisticamente significativa $(p=0,000)$. A pesos menores $(<15 \mathrm{~kg})$ correspondem valores de TBW inferiores a 35L; no entanto, devido ao facto de o tecido adiposo conter menor quantidade de água $(2,25,26)$, não é de desprezar a possibilidade de indivíduos mais pesados exibirem valores mais baixos de TBW.

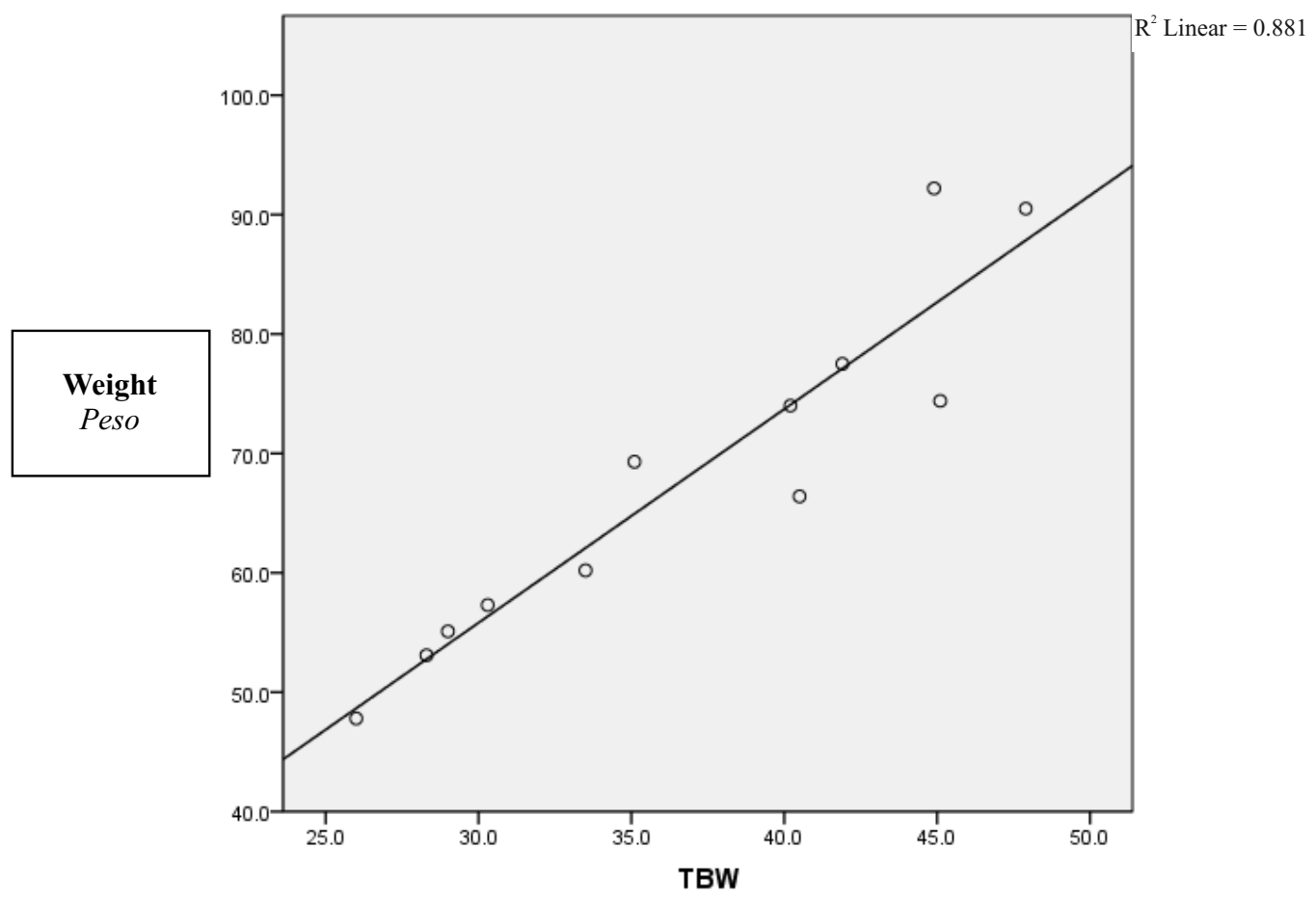

Figure 2 - Graphic representation of data dispersion relating TBW and weight (dots are real values and the line the respective calculated tendency).

Figura 2 - Representação gráfica de dispersão correlacionando as variáveis "TBW" e "peso" (os pontos correspondem aos valores reais e a linha a tendência calculada pela correlação) 


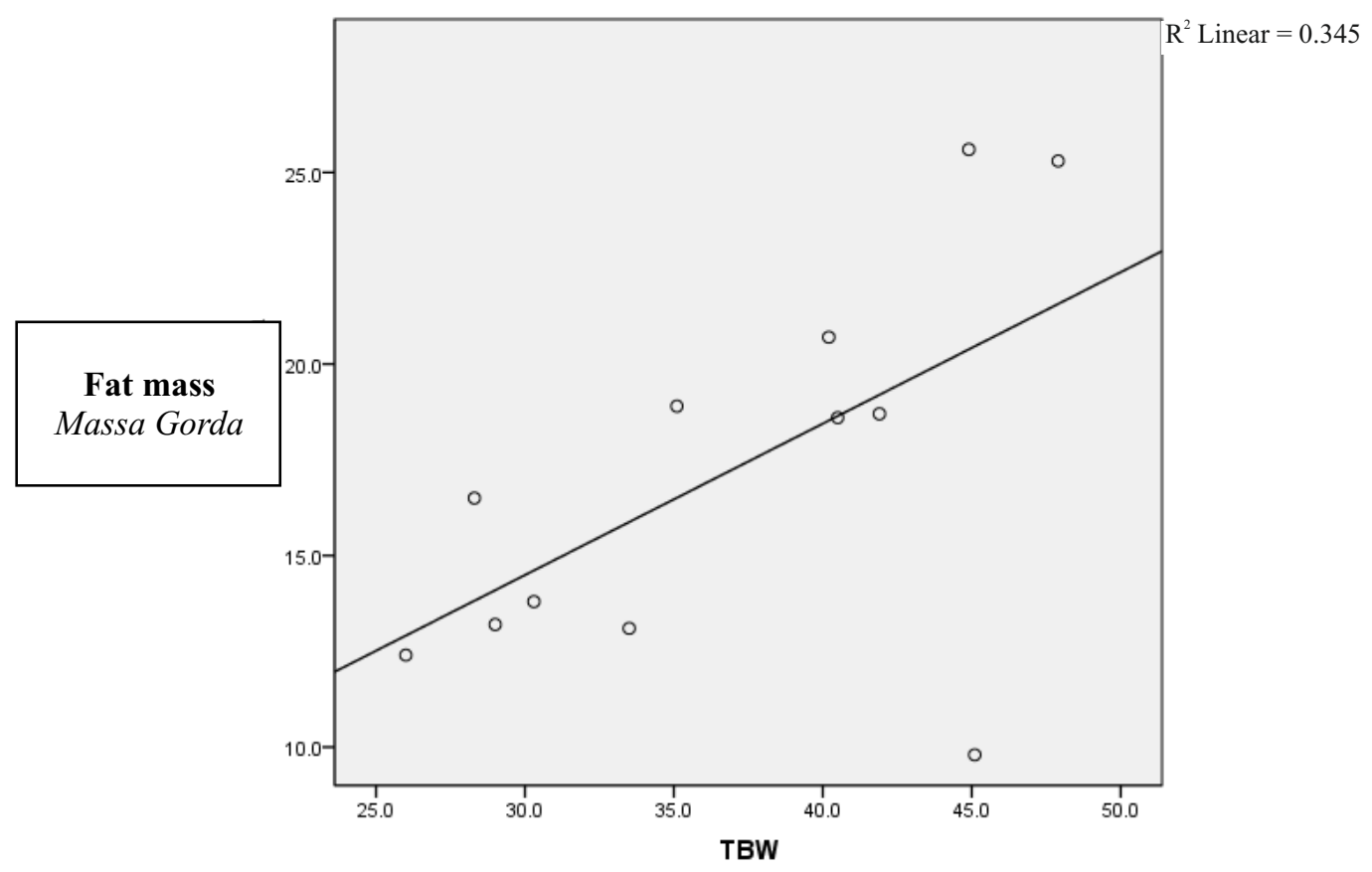

Figure 3 - Graphic representation of data dispersion relating TBW and fat mass (dots are real values and the line the respective calculated tendency).

Figure 3 - Representação gráfica de dispersão correlacionando as variáveis "TBW" e "massa gorda" (os pontos correspondem aos valores reais e a linha a tendência calculada pela correlação)

The same remark should be made regarding TBW and BMI which have shown identical Pearson's correlation coefficients $(\mathrm{Rp}=0,822)$ and significance $(\mathrm{p}=0,001)$.

We have also tried to find significant differences between men and women. As found (Table 1) men exhibit higher mean weight (and range) than women. It is generally accepted that women's weight always involved more "fat mass" (27-29) (which contains lesser water and ion quantities than the so-called "lean mass") than men. This means that, generally speaking, women will have lower TBW than men (Figure 3). The same happens regarding BMI which also shows a relationship with TBW (Figure 3). This reinforces the idea that the mean, in general, will show higher TBW values than for women. Table 4 and Figure 5 illustrate these aspects. Higher TBW values (equal or superior to $40 \mathrm{~L}$ ), belong to men, but when regarding weight, one of the six higher values ( $>60 \mathrm{~kg}$ ), belongs to a woman. This means that it is not possible to establish a direct proportionality rule between weight and TBW. However, in most cases men should show, higher TBW values $\left(r=0,769=\sqrt{ } \mathrm{R}^{2}=\sqrt{ } 0,591\right)$, demonstrating a moderate positive correlation, while women will show lower TBW values $\left(r=0,965=\sqrt{ } R^{2}=\sqrt{ } 0,931\right)$,
A mesma observação é registada para as variáveis TBW e o IMC mostrando idênticos coeficientes de correlação de pearson $(\mathrm{Rp}=0,822)$ e significância $(\mathrm{p}=0,001)$.

Procurámos ainda saber se existiriam diferenças significativas entre homens e mulheres Conforme se verifica (Tabela 1) os homens apresentam um peso médio (e intervalo de valores) mais elevado do que as mulheres. Aceita-se que, em geral, o peso das mulheres envolve uma maior percentagem de massa gorda ${ }^{[27-29]}$ (a qual contém menor quantidade de água e iões relativamente à chamada massa magra) do que o dos homens, pelo que será de prever que as mulheres apresentem, na sua generalidade, valores mais baixos de TBW do que os homens (Figura 3). O mesmo se passa relativamente ao IMC, tendo-se verificado anteriormente (figura 3) que existe uma relação entre TBW e IMC, o que reforça a ideia de que os homens, na sua generalidade, terão valores de TBW superiores aos das mulheres. A tabela 4 e a Figura 5 ilustram estes aspectos. Valores mais altos de TBW (iguais ou superiores a 40L), são todos de homens mas, no que respeita ao peso, dos seis valores de peso mais elevados (superiores a $60 \mathrm{~kg}$ ), um pertence a uma mulher, pelo que não é possível estabelecer uma relação de 
demonstrating a strong positive correlation, as suggested before.

Once the normal distribution of TBW was confirmed, significant differences were found between men and women $(\mathrm{p}<0,001)$, where men presented a higher TBW mean $(43,4 \pm 3,0$ versus $30,4 \pm 3,4)$.

There are published references regarding the quantification of body fluid which relate these ions to intra and extracellular volume compartments ${ }^{[38]}$. Potassium seems to be related to ICW, meaning that low $\mathrm{K}+$ should imply low ICW ${ }^{[22,31]}$. Sodium and chloride are the main components of the extracellular fluid and they seem to be primarily related to the ECW. This would suggest that their variation must be related [22], although some contradiction still exists regarding chloride ${ }^{[32,33]}$. proporcionalidade directa entre peso e TBW. Contudo, admite-se que os homens terão maiores valores de TBW $\left(r=0,769=\sqrt{ } R^{2}=\sqrt{ } 0,591\right)$, sendo a correlação moderada positiva, e que as mulheres terão menores valores de TBW $\left(r=0,965=\sqrt{ } \mathrm{R}^{2}=\sqrt{ } 0,931\right)$, sendo a correlação forte positiva, tal como tinha sido anteriormente sugerido.

Confirmada a distribuição normal da variável TBW, concluímos existir diferenças significativas entre homens e mulheres $(\mathrm{p}<0,001)$ sendo em média os valores de TBW superiores nos homens $(43,4 \pm 3,0$ contra 30,4 43,4 )

No que respeita à quantificação dos compartimentos fluidos, encontram-se descritas na literatura correlações entre os iões aqui estudados e o volume dos compartimentos intra e extracelular ${ }^{[38]}$. O ião potássio parece estar relacionado com a ICW, pelo que, teoricamente, valores baixos de $\mathrm{K}$ deveriam implicar baixo $\mathrm{ICW}^{[22,31]}$; já o ião sódio e o ião cloro, principais electrólitos do fluido extracelular, parecem relacionar-se preferencialmente com a ECW, sugerindo que a sua variação deveria estar relacionada ${ }^{[22]}$, embora relativamente ao ião Cloro existam resultados contraditórios ${ }^{[32,33]}$.

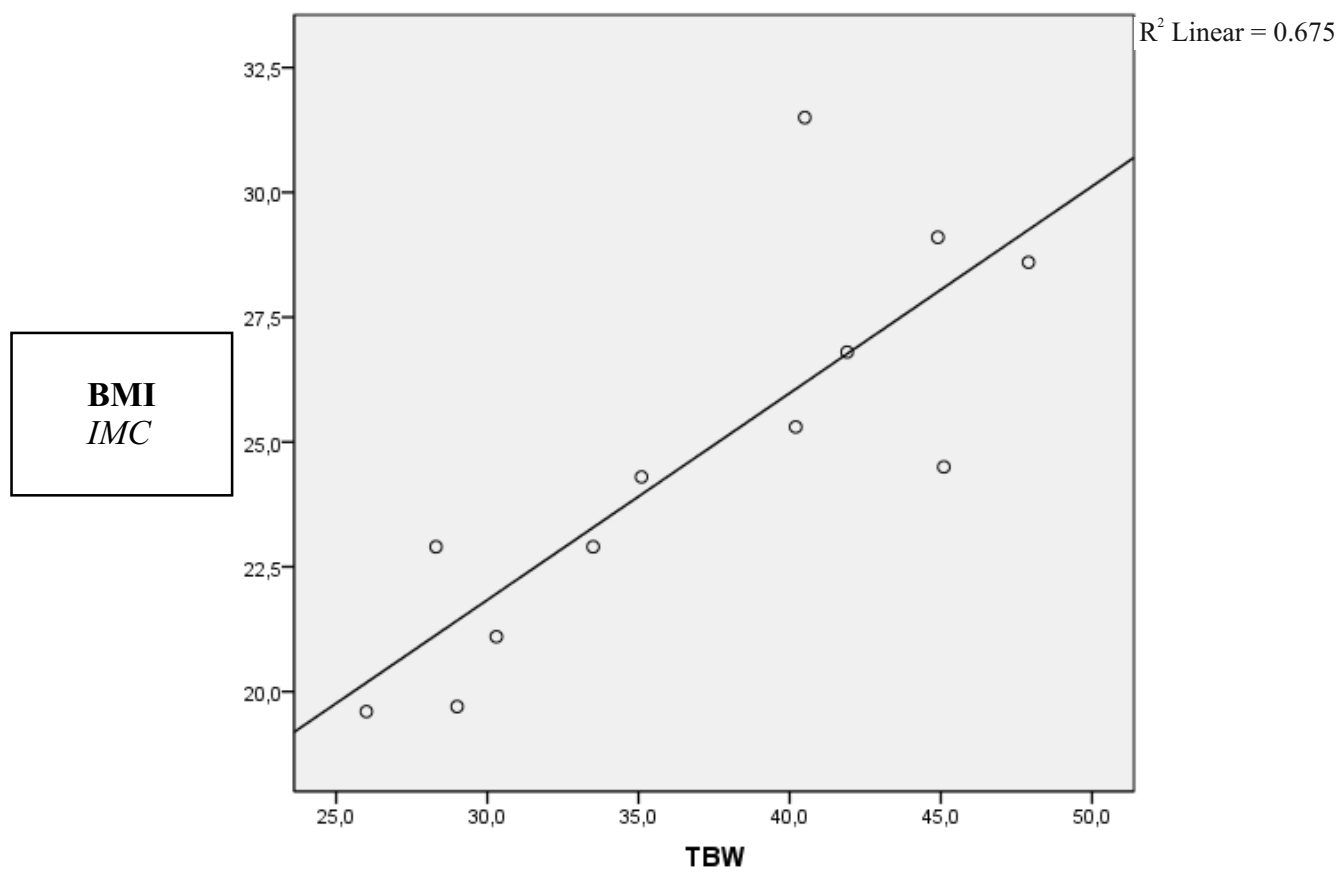

Figure 4 - Graphic representation of data dispersion relating TBW and BMI (dots are real values and the line the respective calculated tendency).

Figura 4 - Representação gráfica de dispersão correlacionando as variáveis “TBW" e "IMC" (os pontos correspondem aos valores reais e a linha a tendência calculada pela correlação) 


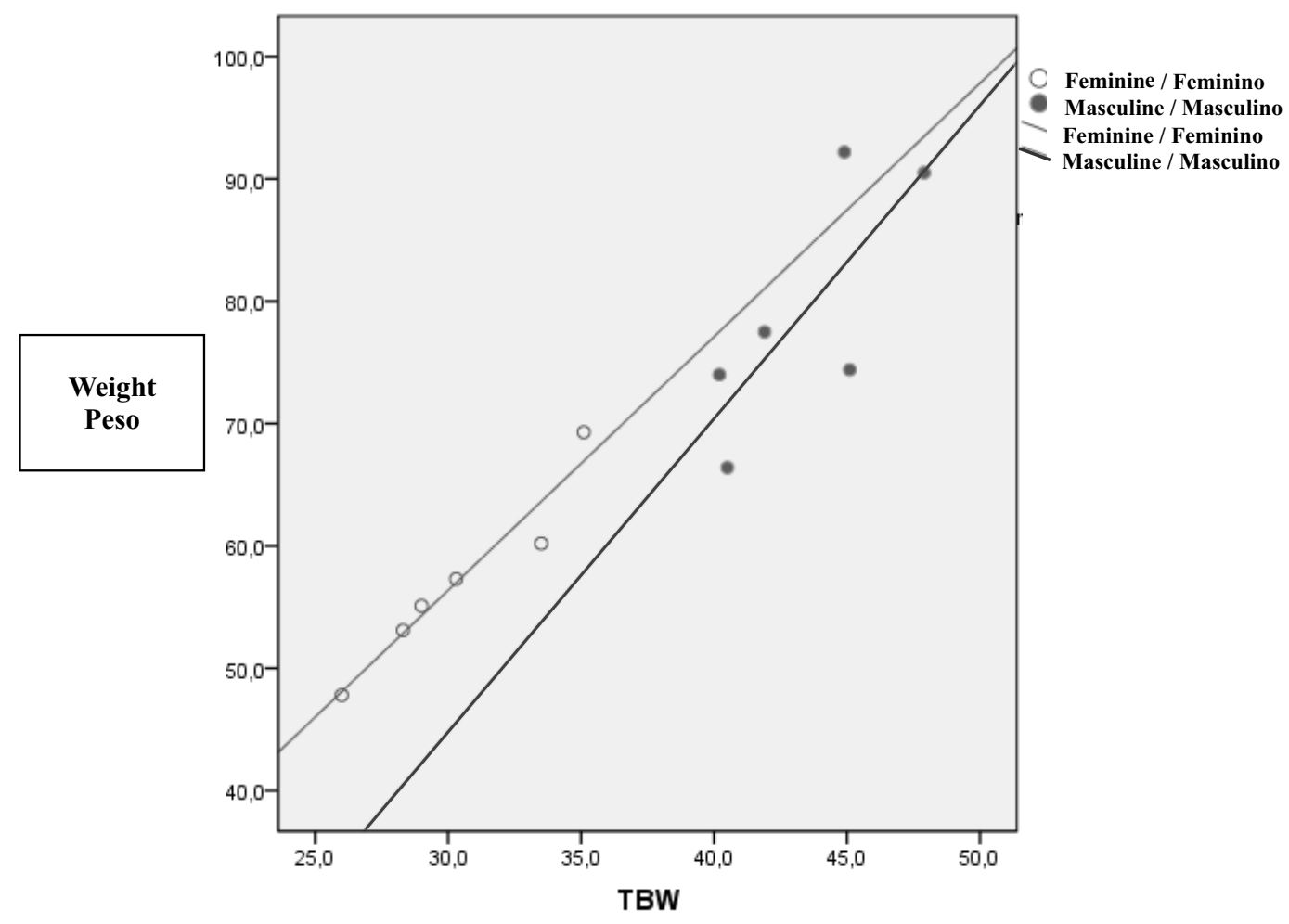

Figure 5 - Graphic representation of data dispersion relating gender, weight and TBW (dots are real values and the line the respective calculated tendency).

Figura 5 - Representação gráfica de dispersão correlacionando as variáveis género, peso e TBW (os pontos correspondem aos valores reais e a linha a tendência calculada pela correlação)

Establishing as the alternative hypothesis "Is ICW value related to the K concentration?" in order to find an eventual correlation between these two variables, the Pearson's correlation analysis was applied, after confirming the variables normal distribution. A statistically significant relation between ICW and $\mathrm{K}+$ variables could not be found $(\mathrm{R} p=0,330 ; \mathrm{p}=0,295)$.

The same rationale was applied to ECW and $\mathrm{Cl}$ variables accepting, as alternative hypotheses "Is ECW value related to $\mathrm{Cl}$ - concentration?" and "Is $\mathrm{ECW}$ value related to $\mathrm{Na}+$ concentration"

No normal distribution was found for ECW and $\mathrm{Cl}$ variables, suggesting that the Spearman's correlation test should be applied. On the contrary ECW and $\mathrm{Na}+$ have shown a normal distribution and a Pearson's correlation test was applied. This analysis could not reveal any sort of relationship between variables (ECW and $\mathrm{Cl} \mathrm{R}=-0,176 ; \mathrm{p}=0,584$; and $\mathrm{ECW}$ and $\mathrm{Na}$ $\mathrm{R}=0,383 ; \mathrm{p}=0,219)$. Under these conditions it is not possible to assume that sodium or chloride variations
Estabeleceu-se como hipótese alternativa "Está o valor de ICW relacionado com a concentração de K?" e para averiguar a existência de uma correlação entre estas duas variáveis, utilizámos a correlação de Pearson, havendo antes confirmado a distribuição normal das variáveis. Esta análise confirmou que não existe uma relação estatisticamente significativa entre as variáveis $\mathrm{ICW}$ e $\mathrm{K}+(\mathrm{Rp}=0,330 ; \mathrm{p}=0,295)$

$\mathrm{O}$ mesmo exercício foi realizado para as variáveis ECW e Cl estabelecendo-se, como hipótese alternativa, "Está o valor de ECW relacionado com a concentração de Cl?" e para as variáveis ECW e $\mathrm{Na}+$ estabelecendose, como hipótese alternativa , "Está o valor de ECW relacionado com a concentração de $\mathrm{Na}+$ ?"

As variáveis $\mathrm{ECW}$ e $\mathrm{Cl}$ não exibiam uma distribuição normal, aconselhando a aplicação da correlação de Spearman, enquanto que escolhemos a correlação de Pearson para as variáveis ECW e $\mathrm{Na}+$ após confirmação prévia da sua normalidade. Esta análise revelou não existir relação entre as variáveis $(\mathrm{ECW}$ e 
will directly influence extracellular water and viceversa.

Regardless of the preliminary approach within this project, these results suggest that the Bodystat ${ }^{\circledR}$ QuadScan 4000 system is potentially interesting concerning the body mass quantification, despite knowing that around $5 \%$ of the population may not be included in the regression equation used by this system ${ }^{[4,7,54-59]}$ (the hydrostatic weighting ranges only $3 \%)^{[4,29]}$. However, regarding the quantification of the intra and extracellular fluid compartments, a relationship between the system's parameters and those ions regarded as reliable indicators, could not be found. In any case, the method's practicability suggests an enormous interest for clinical application, in health and disease, for all reliable information here obtained.

\section{Conclusion}

This preliminary approach does not enable us to arrive at definitive conclusions, especially regarding body water quantification. Regardless of the interest of suggested parameters, we should develop this analysis further by reinforcing the representation aspects involved as well as the methodology.

\section{Aknowledgements}

To the S. João de Deus - Health Group (Nova ERA Clínics) for the clinical and analyical support, and to Farmácia Guardiano (Marinha Grande) for lending the Bodystat $\AA$ QuadScan 4000 system that allowed the present study.

\section{Conflict of interests}

The authors declare that there are no financial and personal relationships that could be viewed as presenting a potential conflict of interests.
Cl $\mathrm{R}=-0,176 ; \mathrm{p}=0,584$; e $\mathrm{ECW}$ e $\mathrm{Na} \mathrm{R}=0,383$; $\mathrm{p}=0,219$ ) não sendo possível assumir, nestas condições, que variações na concentração de sódio, ou de cloreto, se reflectirão na água extracelular e viceversa.

Apesar do carácter preliminar desta abordagem, os resultados aqui obtidos parecem sugerir que o equipamento Bodystat ${ }^{\circledR}$ QuadScan 4000 revela uma interessante aplicabilidade no que respeita à quantificação da massa corporal, mesmo considerando que cerca de $5 \%$ da população poderá não se incluir na equação de regressão utilizada pelo aparelho (4,7,54-59) (na pesagem hidrostática, esta percentagem é de apenas $3 \%)(4,29)$. Contudo, no que respeita à quantificação dos compartimentos intra e extracelular não foi possível identificar uma relação dos parâmetros fornecidos pelo sistema com os iões que considerámos como indicadores fisiológicos daqueles compartimentos. Em qualquer circunstância, são de salientar a rapidez e praticabilidade do método, o que o torna especialmente adequado ao acompanhamento clínico das mais variadas condições de saúde e doença que beneficiem da informação fiável que o aparelho possa fornecer.

\section{Conclusões}

O carácter preliminar do presente estudo não permite conclusões devidamente consolidadas, sobretudo no que respeita à quantificação da água corporal em que, apesar do interesse dos indicadores sugeridos, deveremos ainda procurar esclarecer se esta análise pode ser melhorada através do aumento do número de pacientes ou através da modificação da metodologia de estudo.

\section{Agradecimentos}

Ao S. João de Deus - Grupo de Saúde (Clínica Nova ERA) pelo apoio clínico e analítico recebido, e à Farmácia Guardiano (Marinha Grande) pela amável cedência do equipamento Bodystat ${ }^{\circledR}$ QuadScan 4000 que tornou possível este estudo.

\section{Conflito de Interesses}

Os autores declaram não existir qualquer relação pessoal ou financeira que possa ser entendida como representando um potencial conflito de interesses. 


\section{References / Referências}

1. Grimnes S, Martinsen OG. Bioimpedance and bioelectricity basics. 1st ed. London: Academic Press, 2000.

2. Bodystat. Bodystat ${ }^{\circledR}$ QuadScan 4000 Hardware User's Guide. Isle of Man:2007.

3. Portable Fluids and Electrolytes. Lippincott Williams \& Wilkins; 2008.

4. Total Body Water [cited 6 Feb 2012]. Available from:

http://www.stableisotopeanalysis.com/total_body water.php

5. Armstrong LE. Assessing Hydration Status: The Elusive Gold Standard. J Am Coll Nutr. 2007;26(5):575-84

6. Quinn E. Hydrostatic underwater weighing - how to measure body fat with hydrostatic underwater weighing. [cited 9 Feb 2012]. Available from: http://sportsmedicine.about.com/od/fitnessevaland assessment/g/UnderwaterWeigh.htm

7. Siri WE. Body composition from fluid spaces and density: Analysis of methods. 1961. In: Brozek J and Henschel A (eds.) Techniques for measuring body composition. Washington, DC;National Academy of Sciences:223-44

8. Brozek J, Grande F, Anderson JT, Keys A. Densitometric analysis of body composition: revision of some quantitative assumptions. Ann N Y Acad Sci. 1963:110:113-40.

9. Carvalho ABR, Neto CSP. Composição corporal através dos métodos da pesagem hidrostática e impedância bioelétrica em universitários. Revista Brasileira de Cineantropometria e Desempenho Humano. 1999:1(1):18-23.

10. Miklavcic D, Pavselj N, Hart FX. Electric properties of tissues. Wiley Encyclopedia of Biomedical Engineering. John Wiley and Sons; 2006

11. Kyle UG et al. Bioelectrical impedance analysis - part I: review of principles and methods. Clinical Nutrition. 2004;23:1226-43

12. Wang ZM, Deurenberg P, Wang W, Pietrobelli A, Baumgartner RM, Heymsfield SB. Hydration of fatfree body mass: review and critique of a classic body-composition constant. Am J Clin Nutr. 1999;69(5):833-41.
13. Speakman JR, Visser GH, Ward S, Król E. 3 - The isotope dilution method for evaluation of body composition. In: Body Composition Analysis of Animals - A Handbook of Non-destructive Methods 1st ed. Cambridge:Cambridge University Press; 2011. Chapter 3; p. 56-98.

14. Yuhasz MS. Physical Fitness Manual. London Ontario:University of Western Ontario;1974

15. Levenhagen DK et al. A comparison of air displacement plethysmography with three other techniques to determine body fat in adults. J Parenter Enteral Nutr. 1999:23(5):293-9.

16. MeasureUp. Dual energy X-ray Absorptiometry. [cited 9 Feb 2012]. Available from: http://www.measureup.com.au/body-composition 17. WMA Declaration of Helsinki - Ethical Principles for Medical Research Involving Human Subjects. [cited 9 Feb 2012]. Available from: http://www.wma.net/en/30publications/10policies/b 3/index.html

18. Council of Europe - ETS no. 164 - Convention for the Protection of Human Rights and Dignity of of the Human Being with regard to the Application of Biology and Medicine: Convention on Human Rights and Biomedicine. [cited 9 Feb 2012.] Available from:

http://conventions.coe.int/Treaty/en/Treaties/Html/ 164.htm

19. Wanderley EN, Ferreira VA. Obesity: a plural perspective. Cien Saude Colet. 2010:15(1):185-94. 20. Hannan WJ, Cowen SJ, Plester CE, Fearon KCH, deBeau A. Comparison of bio-impedance spectroscopy and multi-frequency bio-impedance analysis for the assessment of extracellular and total body water in surgical patients. Clinical Science. 1995:89:651-8

21. Mohapatra SN, Costeloe KL, Hill DW. Blood resistivity and its implications for the calculation of cardiac output by the thoracic electrical impedance technique. Intensive Care Medicine. 1977:3(2):63-7. 22. "Regulation of water and electrolyte balance" in "Clinical Physiology of acid-basesd and electrolyte disorders" Rose BD \& Post TW Edts, 5th Edition , McGrw-Hill, NY, 2001,pp239

23. Dehghan M, Merchant AT. Is bioelectrical impedance accurate for use in large epidemiological studies? Nutrition Journal. 2008;7:26.

24. Visser KR. Electric conductivity of stationary and flowing human blood at low frequencies Medical and Biological Engineering and Computing. 1992:30(6):636-40.

25. Evans WD, McClagish H, Trudgett C. Factors affecting the in vivo precision of bioelectrica impedance analysis. Appl. Radiat Isot. 1998 MayJun;49(5-6):485-7.

26. Barnes, C. Body fat scales review and compare [internet]. 2009. Available from:

http://www.choice.com.au/reviews-and-tests/foodand-health/diet-and-exercise/weight-loss/body-fatscales-review-and-compare [cited 9 Feb 2012].

27. Jackson AS et al. The effect of sex, age and race on estimating percentage body fat from body mass index: The Heritage Family Study. International Journal of Obesity. 2002:26(6):789-96.

28. Muth, ND. What are the guidelines for percentage of body fat loss? [cited 9 Feb 2012] Available from:

http://www.acefitness.org/blog/112/what-are-theguidelines-for-percentage-of-body-fat/

29. Gallagher D et al. Healthy percentage body fat rangers: an approach for developing guidelines based on body mass index. Am J Clin Nutr. 2000:72(3):694-701.

30. Walker HK, Hall WD, Hurst JW. Clinical Methods: The History, Physical and Laboratory Examinations [internet]. 3rd ed. Boston: Butterworths; 1990. Chapter 197.

31. Gowrishankar M, Chen CB, Mallie JP, Halperin ML. What is the impact of potassium excretion on the intracellular volume fluid: Importance of urine anions. Kidney International. 1996;50:1490-5

32. Serum Chloride. [cited 9 Feb 2012]. Available from:

http://www.ncbi.nlm.nih.gov/books/NBK309/

33. Nursing Central: Hypochloremia. [cited $9 \mathrm{Feb}$

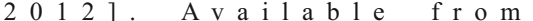
http://www.unboundmedicine.com/nursingcentral/ u b / v i e w / D i s e a s e s - a n d Disorders/73623/all/Hypochloremia 